\title{
CoViCAD: Comprehensive Visualization of Coronary Artery Disease
}

\author{
Maurice Termeer, Javier Oliván Bescós, Marcel Breeuwer, \\ Anna Vilanova, Member, IEEE, Frans Gerritsen, M. Eduard Gröller, Member, IEEE
}

\begin{abstract}
We present novel, comprehensive visualization techniques for the diagnosis of patients with Coronary Artery Disease using segmented cardiac MRI data. We extent an accepted medical visualization technique called the bull's eye plot by removing discontinuities, preserving the volumetric nature of the left ventricular wall and adding anatomical context. The resulting volumetric bull's eye plot can be used for the assessment of transmurality. We link these visualizations to a 3D view that presents viability information in a detailed anatomical context. We combine multiple MRI scans (whole heart anatomical data, late enhancement data) and multiple segmentations (polygonal heart model, late enhancement contours, coronary artery tree). By selectively combining different rendering techniques we obtain comprehensive yet intuitive visualizations of the various data sources.
\end{abstract}

Index Terms-Cardiac MRI, late enhancement, viability, bull's eye plot.

\section{Introduction}

The human heart consists of four chambers; two atria and two ventricles, which are both classified as left and right. The right ventricle pumps the blood through the lungs while the left ventricle pumps the blood through the rest of the body. The muscle tissue that forms the wall of both ventricles is called the myocardium. The part of the myocardium that separates the left and right ventricles is called the septum. As any muscle tissue, the myocardium requires oxygen to operate. This is supplied by the coronary arteries; numerous vessels surrounding the heart.

Over time a stenosis, i.e. a partial or complete occlusion of a vessel, may develop in one or more of the coronary arteries due to various causes. A patient with a stenosis in one or more vessels is said to have Coronary Artery Disease (CAD). If a stenosis continues to develop and eventually completely occludes the vessel, the patient suffers a myocardial infarction, also known as heart attack. Part of the heart muscle tissue experiences a prolonged shortage of oxygen, which causes damage. In some occasions this damage is irreversible. The detection and quantification of such infarcted myocardial tissue, also called scar, is of importance for the assessment of the infarct location and severity. The severity of scar is partially determined by the transmurality; how far the scar extends into the left ventricular wall. Quantification of scar is also important for the selection and planning of therapy such as revascularization via the placement of a stent or a bypass.

Magnetic Resonance Imaging (MRI) has been shown to be an effective diagnosis technique for CAD. Current MRI technology allows the acquisition of high resolution, 3D Whole-Heart (WH) scans that expose mainly anatomical detail. Among the wide variety of MRI acquisition protocols is Late-Enhancement (LE) imaging, a protocol that uses a contrast agent to visualize scar. A LE scan is made 15 to 20 minutes after injection of a contrast agent. Because scar can contain more contrast agent than healthy tissue and experiences a delayed wash in

- Maurice Termeer and M. Eduard Gröller are with the Institute of Computer Graphics and Algorithms, Vienna University of Technology, E-mail: \{maurice|groeller\}@cg.tuwien.ac.at.

- Javier Oliván Bescós, Marcel Breeuwer and Frans Gerritsen are with Philips Medical Systems, E-mail:

\{marcel.breeuwer|javier.olivan.bescos|frans.gerritsen\}@philips.com.

- Anna Vilanova is with the Eindhoven University of Technology, E-Mail: a.vilanova@tue.nl

Manuscript received 31 March 2007; accepted 1 August 2007; posted online 27 October 2007.

For information on obtaining reprints of this article, please send e-mail to: tvcg@computer.org. of the contrast agent, it shows up brighter on the scan than healthy tissue does. LE scans commonly have a much lower resolution than WH scans and thus exhibit fairly little anatomical detail.

Currently the results of MRI-based scar quantification methods are examined using solely $2 \mathrm{D}$ visualization techniques. Apart from examining the individual slices, the bull's eye plot is often used to obtain a schematic representation of the scar in a patient. The bull's eye plot maps the left ventricular wall to a colored circle with the apex being mapped to the circle center. This $2 \mathrm{D}$ visualization technique gives an overview of the entire LE scan and eliminates shape variations of the left ventricle between patients. The approach has two important drawbacks. First it is difficult to make a relation to the 3D anatomy. Even when using the LE slices, all relations have to be done mentally. Second it is impossible to relate scar to specific coronary arteries, which may be the cause of the scar, in a patient-specific way.

We present novel visualization techniques for the diagnosis of patients with CAD that overcome these limitations. We extent the bull's eye plot by unfolding the myocardium and preserving the volumetric nature of the left ventricular wall. This allows for a much better visualization of transmurality and thus assessment of the severity of scar than by using the bull's eye plot. We also present a 3D visualization of scar in an anatomical context that clearly shows the shape and location of the scar in relation to the myocardial anatomy. We annotate this view with various visualizations of the anatomy of the coronary arteries, to relate scar to arteries that may be responsible for supplying the infarcted area. By linking these visualizations together it is possible to navigate through the data examining both myocardial and coronary anatomy and scar shape, location and transmurality in a comprehensive way.

We first discuss related work concerning visualization of cardiac MRI data (section 2). Then an overview of our framework is given along with the data types we use (section 3 ). This is followed by a description of our novel schematic visualization techniques (section 4) and anatomical context visualization (section 5). Afterwards we discuss how a connection between these two visualizations is established through interaction (section 6). Finally, we present the conclusions (section 7)

\section{Related Work}

The American Heart Association has developed a standardized myocardial segmentation model for use among various tomography techniques in cardiovascular science [1]. The model aims at greatly increasing the comparability and reproducibility of diagnoses. Later studies have investigated the correspondence between the model and the coronary arteries [12]. 
Besides the segmentation model a schematic overview to represent the left ventricle in a single diagram was presented. This diagram is called a Bull's Eye Plot (BEP) and consists of a set of concentric circles, which are each divided into a number of segments. The inner circles represent the region near the apex, the bottom of the left ventricle, while the outer circles represent the area near the top of the left ventricle. This diagram is popular in medical practice as it is intuitive and gives a comparable global overview of the property being measured.

Noble et al. [10] combine LE data with information on myocardial contraction to identify regions of the myocardium that suffer from ischemia but are not yet scarred. Such regions can still be revived and identification thereof is thus useful. They propose both the use of a BEP and a projection onto the left ventricular wall as visualization of the resulting parameters. The latter aims to visualize the measurements in an anatomical context, since the BEP is an abstract representation without anatomical information. In their work the outcome was limited, since only a segmentation of the left ventricle was used.

Besides the BEP more visualization methods for the analysis of cardiac tomography data have been proposed, although most of these techniques have not yet been accepted in medical practice. Kuehnel et al. [7] designed software assistants for the analysis of cardiac CT and MRI data. Their approach aims to correlate different measurements by using advanced visualization techniques. In the same context Oeltze et al. [11] presented various visualization techniques for the analysis of perfusion data. Here an integration of visualizations of perfusion measurements with anatomical information is proposed. This allows perfusion defects to be easily related to coronary artery branches feeding the affected area.

Both these approaches propose the use of schematic visualization techniques such as the BEP in combination with $3 \mathrm{D}$ visualization techniques to visualize the data in an anatomical context. The motivation behind this approach is that the reproducibility of the diagnostic work flow is increased, as the relation to anatomy is maintained. However, the schematic visualizations presented offer only little anatomical context, making it hard to relate them to the $3 \mathrm{D}$ visualizations. Moreover, the 3D visualizations do not contain the same information as the schematic views. This requires the data to be combined mentally.

In general medical visualization techniques can greatly benefit from segmentation information. Recently fully automatic segmentation of whole heart MRI data has become possible. The approach presented by Ecabert et al. [2] uses a geometric heart model that can be adjusted to each patient without user interaction. Similar approaches have been developed simultaneously [9]. Full heart segmentations allow a more robust approach to selective visualization, where irrelevant parts of the data are left out. They also provide excellent anatomical context for advanced $3 \mathrm{D}$ visualization techniques that combine various data sources.

\section{Overview of Our Approach}

We have developed a comprehensive visualization framework for the diagnosis of diseased patients suffering from CAD. Our techniques are intended for the combined analysis of LE and WH MRI data, supplying tissue viability and anatomical information, respectively. The MRI scans we used we scanned using protocols common in clinical practice. Our framework consists of both schematic techniques for quantitative analysis and 3D visualization techniques providing anatomical context. The advantage of our approach is that our framework also supplies anatomical context in the schematic visualizations and it is easy to relate the two visualization by interactive exploration.

Our approach of visualizing scar tissue relies on segmentations of both the WH and LE scans. From the WH data we extract a fully automatically generated geometric heart model using a techniques presented by Ecabert et al. [2]. The heart model is a polygonal mesh consisting of 11 parts. It includes the four chambers, the four valves, the aorta, the pulmonary artery and a point indicating the apex. The long and short axes are extracted from the heart model. These two axes are more commonly used for image acquisition than the standard patient axes. Figure 1a shows the intersection of the model with a four-

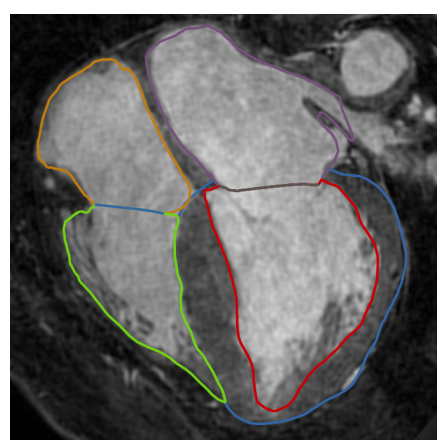

(a)

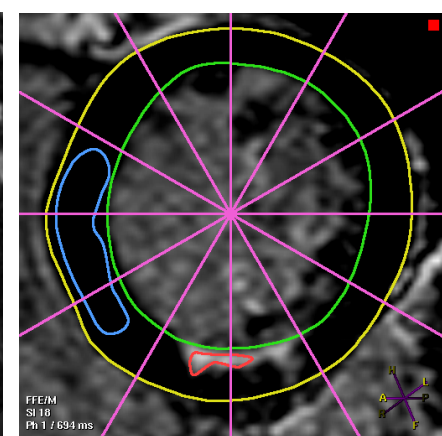

(b)
Figure 1: (a) A four-chamber long-axis view on a WH scan annotated with the heart model intersection lines and (b) a LE slice with manually drawn contours (green and yellow), healthy region (blue) and scar region (red)

chamber long-axis view of a WH scan. Additionally we use a currently experimental prototype [8] to extract the centerlines of the main coronary arteries. This segmentation information captures the shape, size, position and orientation of all the relevant parts of the heart.

Our segmentation of the LE data consists of a set of manually drawn contours of the left ventricle. Endo- and epicardial contours are drawn by the user on each short-axis LE slice where these structures are visible. An example of a LE slice and such manually drawn contours is given in Figure 1b. These contours are then interpolated to form a 3D polygonal mesh. This mesh, which typically does not include the apex, forms a segmentation of the relevant parts of the left ventricular myocardium.

Besides a segmentation, a proper classification of LE data requires a method to discriminate scar from healthy tissue. This is commonly accomplished with the definition of a function that classifies density values as either scar or viable tissue. This scar classification function is defined by the user. Inside the manually drawn contours, the user specifies two regions; one that clearly represents healthy tissue and one that clearly represents scar. These two regions are shown in blue and red respectively in Figure $1 \mathrm{~b}$. For both regions, $[\mu-2 \sigma, \mu+2 \sigma]$ is used as the density range that represents either healthy tissue or scar. In this formula $\mu$ denotes the mean density and $\sigma$ denotes the standard deviation. In case these two intervals do not overlap, a linear transition is defined on the interval $\left[\mu_{\text {healthy }}+2 \sigma_{\text {healthy }}, \mu_{\text {scar }}-2 \sigma_{\text {scar }}\right]$.

The relations between the various data sources and the relation to our analysis system are depicted in Figure 2. The CoViCAD framework can be divided into two visualization components. The first component provides a schematic view of the LE data. It abstracts from the anatomy to give a clear, global overview of the distribution of scar. The second component is the anatomical view; a $3 \mathrm{D}$ view of the heart, visualizing scar tissue in an anatomical context. These two components are coupled by an interaction module that provides an intuitive relation between the two views. The schematic view is intended for the visualization of the overall distribution and transmurality of scar, while the anatomical view is intended for visualizing the size and location of scar in an anatomical context.

\section{The Volumetric Bull's Eye Plot}

The left ventricular myocardium has a complex shape. Not considering the apex, it has approximately the shape of a cylinder. It is however not perfectly circular and the wall thickness varies throughout the entire left ventricle. These properties, as well as the general shape, size and orientation, differ for each patient. The Bull's Eye Plot (BEP) was developed to allow for a structured and reproducible analysis that compares well between different patients. The BEP is a visualization technique that is currently commonly used in clinical environments.

The BEP is a visualization concept that can be applied to various measurements on the left ventricle. Here we focus on viability information provided by LE data. A BEP is in essence a mapping of the 


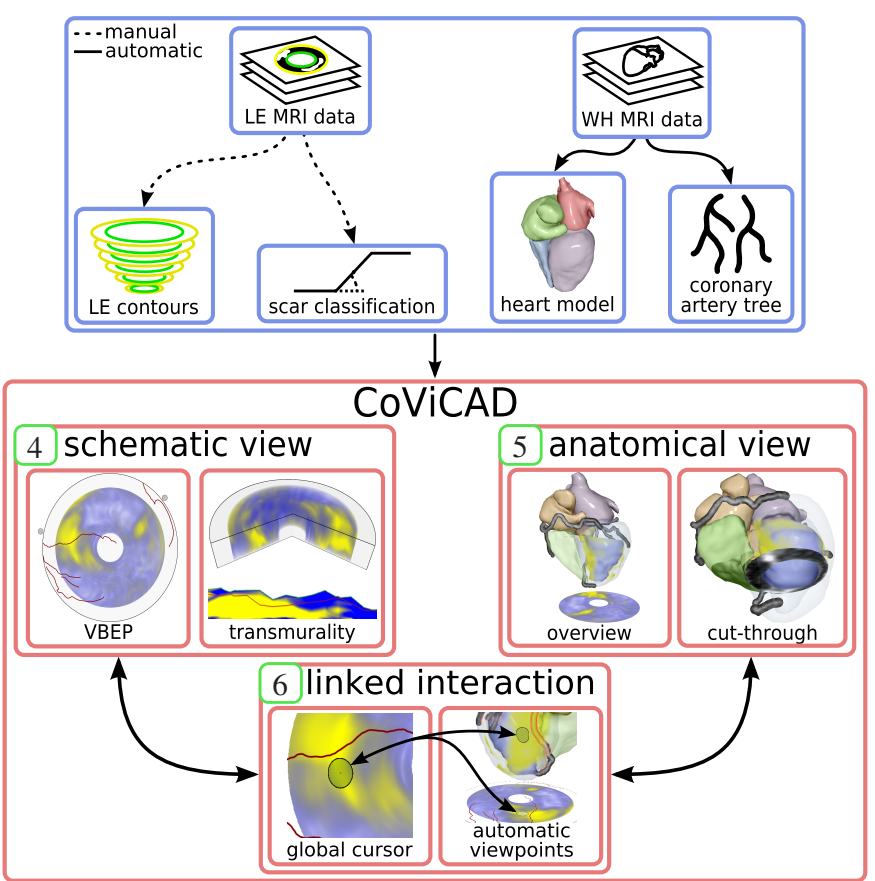

Figure 2: Overview of the CoViCAD framework with the various data sources outlined in blue and the visualization modules outlined in red. The green boxes refer to the sections where the corresponding components are discussed.

myocardium onto a set of concentric rings. For each slice of the LE scan, the segmented myocardium as shown in Figure $1 \mathrm{~b}$ is reformatted to a ring. These rings are combined as a set of concentric circles, with the apex in the middle. An example of a BEP of LE data is shown in Figure 3a. The reformatting is commonly accomplished by dividing the myocardial area of each slice into a fixed set of sectors. For each of these sectors the degree of enhancement is computed and the corresponding sectors in the BEP are colored using a color encoding.

While this approach does eliminate all the inter- and intra-patient shape variations of the left ventricle, it has three main drawbacks. First the quantization of the data into sectors gives a less accurate view than a continuous one. Even though various degrees of quantization are being used, the BEP is discontinuous at the borders between the rings of the different slices. The second, more severe drawback is that it provides no information on the distribution of scar tissue along the ventricle wall; the transmurality of scar. This problem is sometimes solved by displaying two BEPs simultaneously; one that visualizes the amount of scar and one that visualizes the transmurality of scar. The third drawback is that it provides no anatomical information. The BEP is a 2D abstract representation of the LE data, but relating it to anatomical information is necessary. Even with an additional view that provides anatomical information, the relation between these two views would have to be done mentally.

\subsection{Unfolding the Myocardium}

To overcome these limitations, we propose the Volumetric Bull's Eye Plot (VBEP); a 3D extension of the BEP that is continuous, provides transmurality information and is annotated with anatomical information. An example of a VBEP is shown in Figure 3b. It solves the three main disadvantages of the BEP described earlier.

The VBEP is an unfolding of the myocardium along the long axis that is reformatted into a cylinder. This is illustrated in Figure 4. The bottom of the cylinder corresponds to the epicardium and the top corresponds to the endocardium. Thus the volumetric nature of the left ventricular wall is preserved. Like in the BEP, the contours are reformatted to concentric rings to mask any shape variations. There are however three important differences, that solve the three aforementioned disadvantages of the BEP.

The first difference is that, as shown in Figure 4, the distance be- (a)

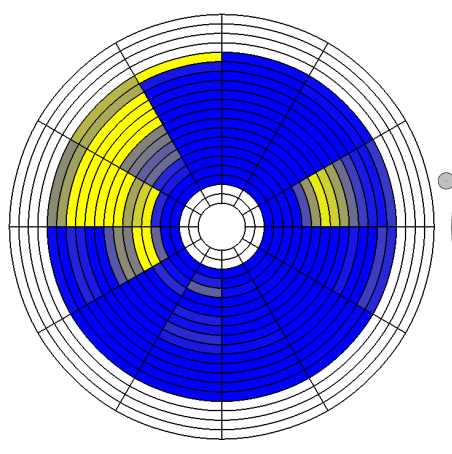

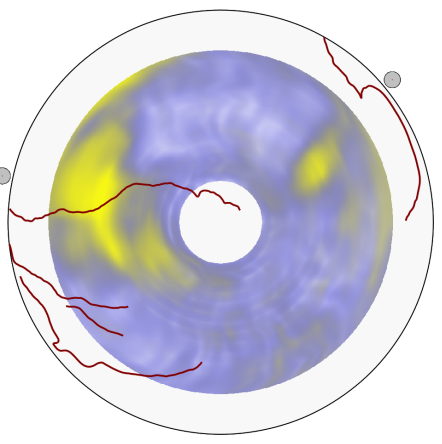

(b)
Figure 3: A comparison between (a) a BEP and (b) a VBEP. The rings in (a) represent the reformatted contours. These are not present in (b), as the VBEP is a continuous volume. The empty rings originate from LE slices for which no contours were drawn, because the myocardium was not present or could not be identified on the corresponding slices. In both figures blue corresponds to healthy tissue and yellow corresponds to scar.

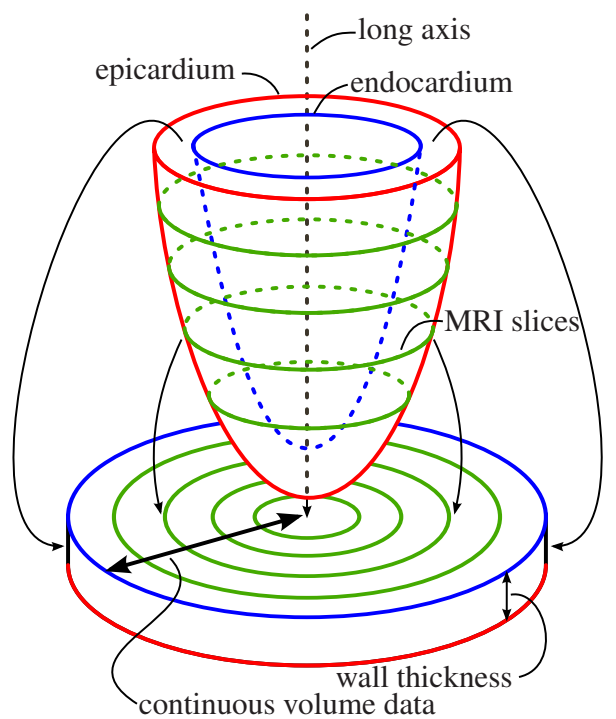

Figure 4: A VBEP is constructed by unfolding and reformatting the myocardium to a cylinder, where the volumetric nature of the ventricle wall is preserved.

tween the epicardium and endocardium is mapped to the thickness of the cylinder. This means that there are no discontinuities between the contours and that the VBEP is a continuous volume. Therefore there is also no need for quantization.

The second difference is that the volumetric nature of the left ventricular wall is preserved. This means that the transmurality information, which is lost in the BEP, is preserved in the VBEP.

The third difference is the addition of anatomical information. As can be seen in Figure 3b, the coronary arteries are projected on top of the epicardial side of the VBEP. This allows scar to be related to coronary arteries. It also allows the orientation of the VBEP to be perceived with respect to the heart. For further perception of orientation, two dots are drawn on the side of the VBEP that correspond to the locations where the right ventricle joins the left ventricle.

The unfolding of the myocardium is realized by computing two 2D transformation maps; one for the epicardium and one for the endocardium. Each transformation map contains at each location the coordinates of the corresponding point in unfolded space. Using transformation maps does not require to resample the volume data into an unfolded representation. Apart from being more memory efficient, using transformation maps also does not introduce interpolation artifacts. For the VBEP each transformation map is a circle that stores 
the coordinates of the endo- or epicardial layer for each point in that circle. Linearity is assumed between the endo- and epicardial layers. Therefore only two 2D maps are required instead of a full 3D map.

This linearity is assumed because each LE contour is reformatted to a circle in the VBEP and the contours have the same spacing as in the LE scan. This is also shown in Figure 4. While it may seem better to unfold along the normal direction of the center surface (in between the endo- and epicardial layers), our choice preserves the relation between the radius dimension in the VBEP and the long axis. In other words, all points on a concentric cylinder in the VBEP lie in a plane orthogonal to the long axis. This is an important and intuitive relation, which is also present in the BEP.

\subsection{Overall Distribution of Scar}

When the cylinder that is the result of the VBEP unfolding is viewed from the bottom, it gives a similar global overview as a BEP. This can be seen by comparing Figures $3 \mathrm{a}$ and $3 \mathrm{~b}$. The volumetric nature of this cylinder requires a suitable accumulation scheme such as Direct Volume Rendering (DVR) or Maximum Intensity Projection (MIP). In Figure $3 \mathrm{~b}$ a DVR strategy is applied. A linear gradient of two visually distinct colors is used to color code the degree of scar according to the scar classification function. In Figure 3 we use blue and yellow representing healthy tissue and scar, respectively. More colors provide little advantage and may cause visual clutter, especially when more annotations are added.

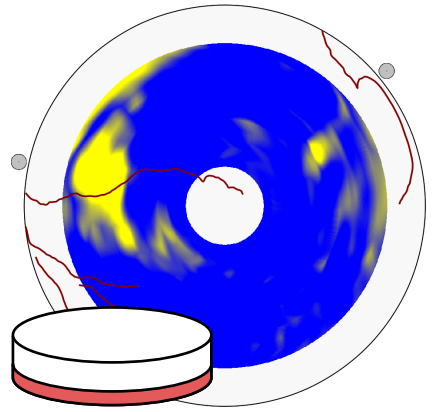

(a)

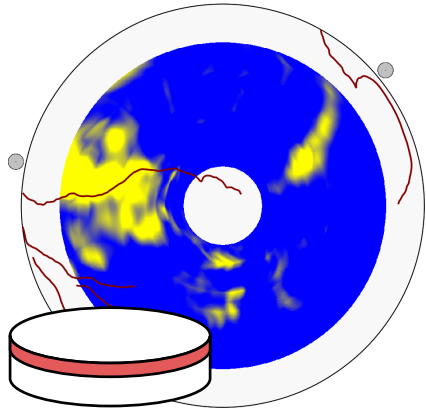

(b)
Figure 5: Two VBEPs that show only a slab of the cylinder; (a) shows only viability information near the epicardial layer and (b) only near the endocardial layer.

In Figure 3b, all the data from the myocardial segmentation is used, similar to the BEP. However, the additional dimension adds more freedom. By selecting a slab of the cylinder, the amount of data visualized in the VBEP can be controlled. This concept is demonstrated in Figure 5. The slab of the cylinder that contains the data represented in the VBEP can be varied. In this way the viability information either near the epicardial surface (Figure 5a), the endocardial surface (Figure 5b) or in the center can be visualized separately. This additional degree of freedom is useful for assessing the severity and to some extent the transmurality of local scar.

\subsection{Transmurality of Scar}

The main advantage of the VBEP is that the thickness of the cylinder contains the transmurality of scar, i.e. the extent of scar tissue into the left ventricular wall. This is demonstrated in Figure 8a, where the VBEP is shown from the side with a wedge cut-out. Along the edge of the cut-out, the transmurality can be observed. However, only a small part of Figure 8a actually shows the transmurality of the region of interest. The cylindrical shape of the VBEP also makes it difficult to assess the transmurality of a larger region of interest.

Therefore we propose a second unfolding of the myocardium specifically intended for exploring the transmurality of a region of interest. The VBEP is an unfolding of the myocardium along the long axis. For the second unfolding, called the rectangular unfolding, the myocardium is first cut open along the long axis and is then unfolded

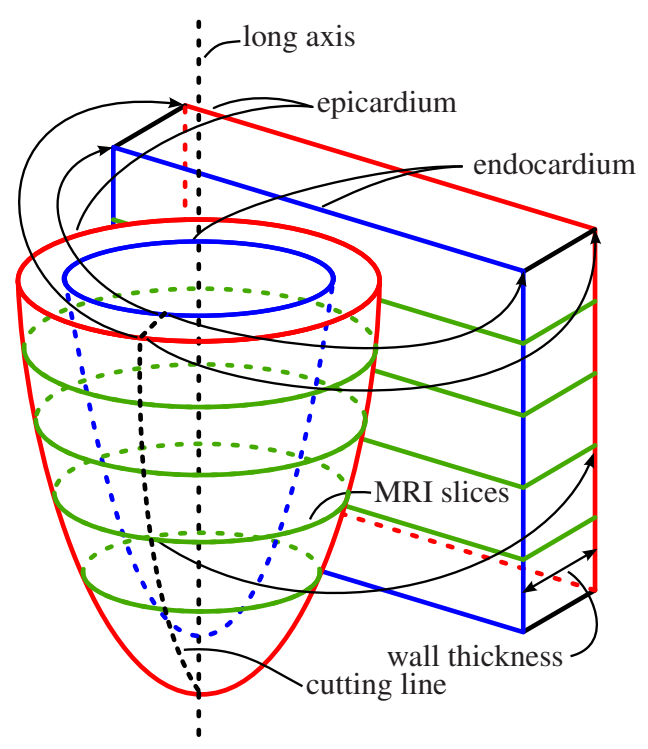

Figure 6: The rectangular unfolding is constructed by cutting open the myocardium along a line parallel to the long axis and unfolding it to a rectangle.

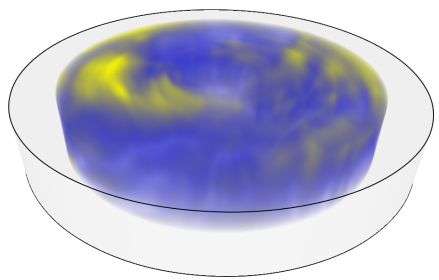

(a)

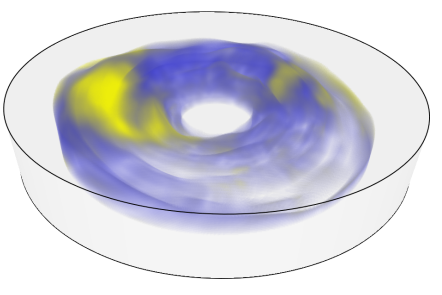

(b)
Figure 7: Wall thickness preservation in the VBEP; (a) uniform wall thickness; (b) absolute wall thickness preserved.

and reformatted to a rectangle. This is depicted in Figure 6. Proportions inside the myocardium are better preserved in the rectangular unfolding than in the VBEP since less stretching is involved during the reformatting. The rectangular unfolding is implemented similar to the VBEP unfolding, only here the transformation maps are rectangular instead of circular. When the rectangularly unfolded myocardium is viewed from the side, the transmurality can be perceived easily.

The motivation behind constructing this rectangular unfolding is as follows. On the VBEP the user can select a pie-shaped area of interest (Figure $8 \mathrm{~b}$ ), for which a visualization of the transmurality is desired. The key observation is that this pie-shaped area in the VBEP corresponds to a rectangular slab in the rectangular unfolding. By visualizing only this slab and viewing it from the side, the transmurality of the region of interest can be observed (Figure 8c). When the pie-shaped section would be viewed from the side, distortions would appear due to variations in thickness along the viewing direction and due to its cylindrical shape. These distortions are no longer present in the side projection of the rectangular slab.

Similar to the VBEP, this approach yields a 3D object and thus requires a suitable accumulation scheme. Figure $8 \mathrm{c}$ demonstrates both a MIP and a DVR scheme. Since the transmurality varies throughout the ventricle, it varies along the viewing direction of the slab. To obtain an accurate visualization of the transmurality, the slab should remain thin. Thus the pie-shaped area on the VBEP should not be too big, as this may occlude the variations in transmurality inside that area. For example, with a DVR scheme only the transmurality of the front part would be visible, while with a MIP scheme only the maximum transmurality of the slab is visualized. 


\subsection{Preservation of Wall Thickness}

In the VBEP the 3D nature of the left ventricular wall is preserved. In Figure 4 the wall thickness was reformatted to be uniform. The absolute wall thickness information can also be preserved. This would cause the height to vary throughout the VBEP, as is demonstrated in Figure 7. Figure 7a shows a VBEP with uniform thickness. In Figure $7 \mathrm{~b}$ the height of the cylinder was scaled at each location using the absolute wall thickness at that location and the difference between the maximum and minimum wall thickness. The projection used in Figure 7 is for demonstration purposes only. The rectangular unfolding captures phenomena inside the ventricle wall in a better way.

When the myocardium is reformatted to have uniform thickness, this allows for the perception of relative transmurality (\%). When absolute wall thickness information is preserved, it allows for perception of absolute transmurality ( $\mathrm{mm})$. This distinction is relevant during diagnosis. Relative transmurality gives a more uniform visualization of the overall distribution of scar, not distorted by the varying wall thickness. In the case of partial transmurality, the absolute thickness of the remaining healthy tissue is relevant for the estimation of gained functionality if it would undergo revascularization.

The absolute wall thickness information is of most value when assessing the transmurality of scar. While top or bottom projection of the VBEP gives an overview of the distribution of scar, absolute wall thickness information and transmurality are best visible in the rectangular unfolding. Wall thickness is preserved in the rectangular unfolding in an identical way as for the VBEP. Figure 8c shows an example of a transmurality visualization both with uniform and with absolute wall thickness information. We visualize the wall thickness by adjusting the distance of the endocardial contour to the epicardial contour. This keeps the epicardial side flat and eases comparison of wall thickness throughout the slab.

The wall thickness may vary throughout the slab along the viewing direction. We visualize this variation using lines indicating the minimum and maximum wall thicknesses. These lines can be used to detect large variations in wall thickness in the slab. If the two lines are far apart, variations in wall thickness are causing an inaccurate visualization of transmurality and a thinner slab should be selected. This is similar to the phenomenon that a too thick slab occludes transmurality due to the accumulation scheme as discussed in section 4.3.

\subsection{Overlaying Coronary Arteries}

As additional context information, the coronary arteries are projected on the epicardial surface of the VBEP. We first project each vessel centerline to the epicardial surface. Next these centerlines on the epicardial surface are mapped to the corresponding centerlines on the VBEP using the same unfolding. The projection is necessary since the unfolding transformation is only defined within the volume of the LE contours. It can generally be assumed that each part of the myocardium is fed by the closest coronary artery. The projection of the coronary arteries on the epicardial surface has no significant impact on the relation between the myocardial tissue and its closest coronary artery. It is therefore an acceptable solution. Parts of the coronary arteries that have a distance to the epicardial layer greater than a preset threshold, e.g. coronary arteries feeding the right ventricle, are not projected onto the VBEP.

An example of a VBEP with the coronary arteries projected on the epicardial surface is given in Figure $3 \mathrm{~b}$. The coronary arteries act as additional context information only and no diameter information is available. They are rendered as lines with a single color and without shading information to avoid visual clutter as much as possible.

\section{Viability in an Anatomical Context}

The VBEP has more anatomical context than the BEP, but it still is an abstraction from the actual anatomy. The anatomical view of the CoViCAD framework provides a 3D view of the heart intended for the observation of viability information in an anatomical context. This anatomical context is especially useful for the observation of location and size of scar. Opposed to the schematic view of our framework, the shape and proportions of the structures in the heart are preserved.

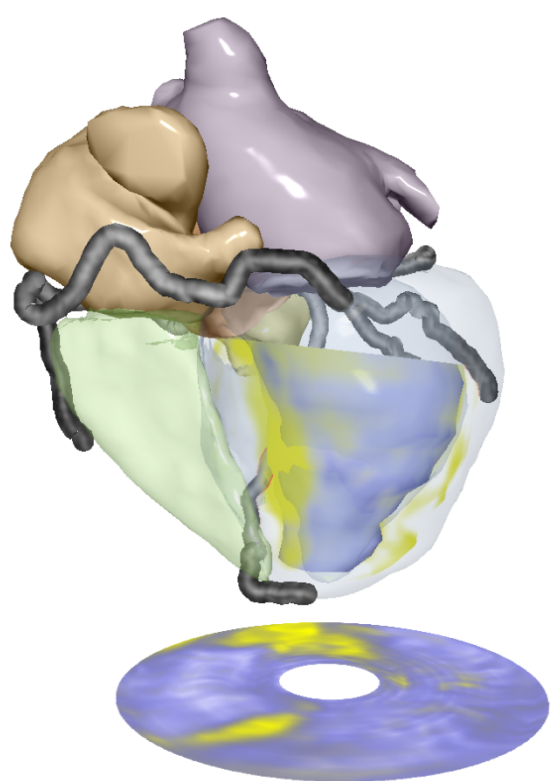

Figure 9: Anatomical view showing the individual parts of the heart, the coronary artery tree and scar tissue in yellow inside the left ventricular myocardium.

While similar approaches of visualizing viability information in an anatomical context have been presented before [10,11], our approach differs in that we combine multiple data sources with multiple visualization techniques. This approach has been shown to be useful before [4]. We use detailed anatomical information extracted from the WH scan. We combine a volume rendering of the LE data with the geometric heart model. Additionally we render the coronary artery tree as a set of solid tubes, as a MIP or as a Curved Planar Reformation (CPR) [5] of the neighborhood of the vessel centerline. This makes it possible to relate scar to a coronary artery branch.

An example of our anatomical view is shown in Figure 9. The LE data is rendered with Direct Volume Rendering (DVR). All LE data is rendered using a single constant color, only the opacity varies based upon the scar classification function. In Figure 9 scar is visible as the yellow areas in the wall of the left ventricle. The epicardial wall is rendered semi-transparently to prevent occlusion of the LE data. The endocardial wall is rendered in opaque blue so only scar in the front is visible.

We use depth peeling [3] on a closed polygonal mesh constructed from the manually drawn contours to ensure that only the LE data inside the myocardium is rendered. This mesh is typically concave and an approximate solution that assumes convexity is not acceptable. The myocardium is surrounded by other bright structures that could easily be misinterpreted as scar. The depth layers extracted with depth peeling, are used to determine which part of each viewing ray intersects the myocardium. We typically use four depth layers.

Since the LE data provides little anatomical detail, we display the geometric heart model and the coronary artery tree, both extracted from the WH data, as additional anatomical context information. For contextual purposes this segmentation provides sufficient detail and this visualization would not benefit from adding the original MRI data with e.g. DVR. The original MRI data should however still be accessable to the user. The individual parts of the heart model are rendered in visually distinct colors to make them visually clearly separable. The same strategy is applied to the coronary artery tree. Shading is applied using the Phong shading model for increased perception of shape. For navigational purposes a VBEP of the LE data is displayed on the ground plane. It serves as a map of the LE data and gives a better perception of the orientation of the heart.

\subsection{Visualization of Coronary Arteries}

The depiction of the coronary arteries in the anatomical view is of great diagnostic value. Once a region of scar is identified, it can be related 


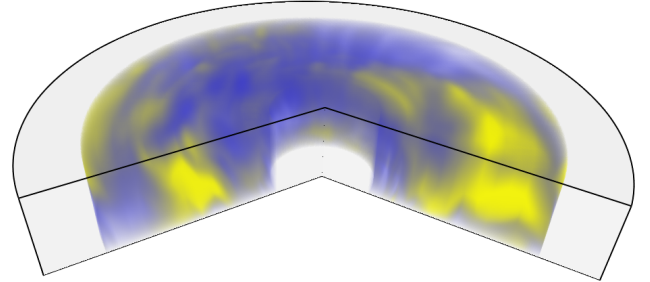

(a)

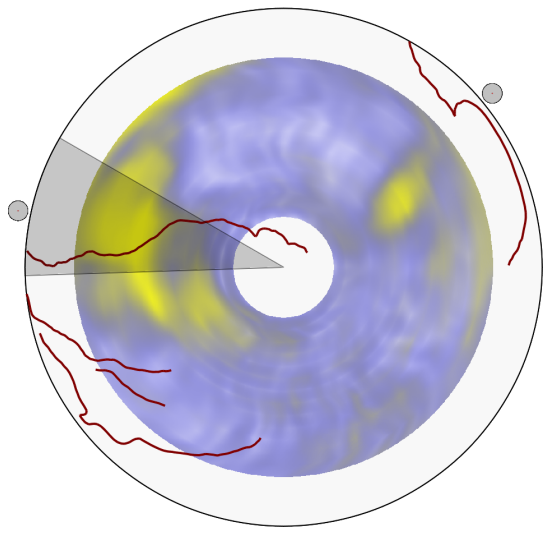

(b)
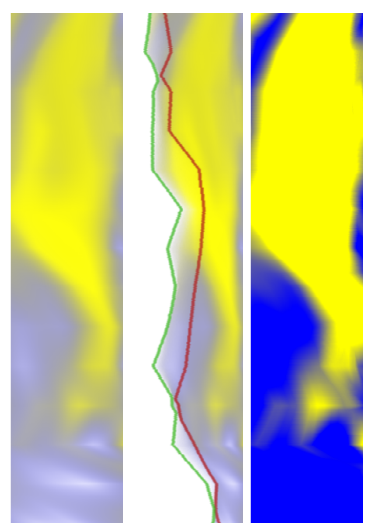

(c)

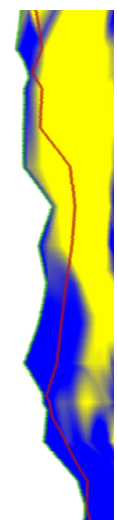

Figure 8: Visualizing transmurality using the VBEP: (a) a VBEP with a wedge cut-out; (b) a VBEP with a region of interest highlighted and (c) a DVR (left two) and MIP (right two) of the region of interest viewed from the side, exposing the transmurality of the scar, both without (left) and with (right) absolute wall thickness information. The red and green lines correspond to the minimum and maximum wall thicknesses of the region of interest, respectively.

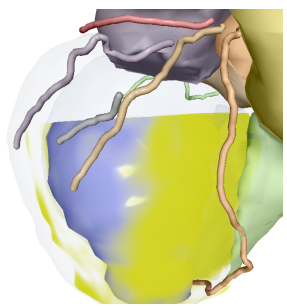

(a)

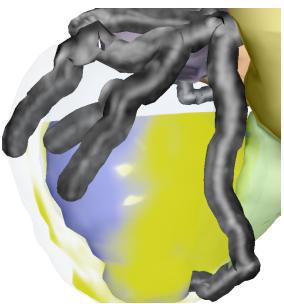

(b)

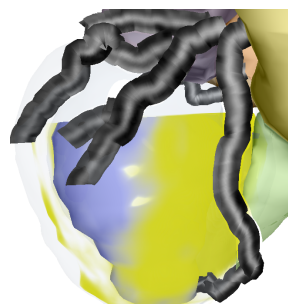

(c)

Figure 10: The coronary arteries rendered as (a) polygonal tubes of constant radius around the vessel centerline; (b) as a MIP of the WH data inside the tubes and (c) as a CPR of a surface through the tubes.

to a coronary artery near that area. The segmentation we use only provides the centerline of the vessels. Current MRI technology does not provide enough resolution to extract absolute diameter information for the coronary arteries [6]. This also means that surface rendering techniques will not provide accurate results. The image data can however provide an indication of a possible stenosis, which can then be further investigated using other technologies such as angiography.

To facilitate the detection of a possible stenosis, we offer three different visualizations of the coronary arteries. For each vessel, a polygonal tube around its centerline is constructed. These tubes have a constant radius that can be specified by the user. The first visualization of the arteries renders these tubes in visually distinct colors (Figure 10a). The tubes are rendered with a small radius, to avoid the false impression of giving vessel diameter information and to cause minimal occlusion. This representation allows scar to be related to a particular coronary artery.

The second visualization is a Maximum Intensity Projection (MIP) of the WH data inside the tube (Figure 10b). This approach requires a larger diameter of the tube, since the lumen of the vessel should be clearly distinguishable. The localized MIP allows perception of the vessel diameter and detection of a possible stenosis. Using a MIP preserves the 3D nature of the vessels and is thus an intuitive representation. A downside is that when the radius of the tube is made sufficiently large, neighboring structures might be included in the rendering and may occlude the vessel.

The third representation of the coronary arteries does not suffer from this drawback. It uses a Curved Planar Reformation (CPR) [5] along a ruled surface through the centerline (Figure 10c). The combination of volume rendering with CPR has been presented before [13]. An initial orientation of the surface used for the CPR is computed using the long axis extracted from the heart model. The vector from a

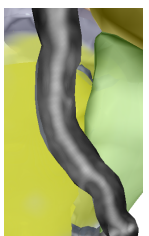

(a)

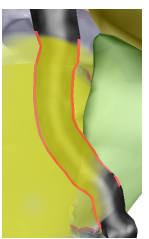

(b)

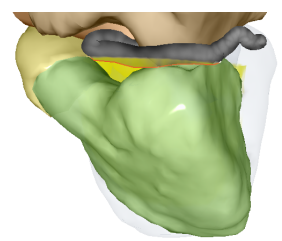

(c)

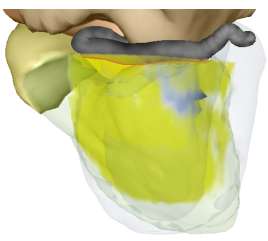

(d)
Figure 11: Solving occlusion issues: (a) coronary artery occluding scar; (b) coronary artery rendered semi-transparently with outline highlighted; (c) right ventricle occluding scar in the septum and (d) revealing the septum by making the right ventricle semi-transparent.

point on the vessel centerline to the corresponding closest point on the long axis is taken as the normal vector of the CPR surface through that point. This causes the surface to be approximately tangential to the epicardial surface near the vessel. The user can specify an offset angle that controls the rotation around the centerline of the vessel.

A different surface is used for displaying the reformation to ensure that the CPR is always well visible. This display surface is similar to the reformation surface, except that it is always oriented towards the viewer. This has the advantage that changing the viewpoint does not change the CPR representation of the coronary arteries. While the CPR has the advantage that the vessel lumen is always visible, it does not give a $3 \mathrm{D}$ view on the vessel neighborhood, contrary to the MIP rendering.

\subsection{Resolving Occlusion of Scar}

To prevent occlusion of the LE data by the coronary arteries, the opacity of the coronary arteries can be locally controlled by the opacity of the LE data it is occluding. We call this coronary ghosting. Figures $11 \mathrm{a}$ and $11 \mathrm{~b}$ demonstrate this concept. The degree of transparency is determined by the amount of scar that is visible beneath the artery. Coronary arteries occluding areas that contain no scar are rendered fully opaque. Areas with heavy scarring, such that it shows up completely opaque in the volume rendering, cause the coronary arteries to be rendered with a very low opacity. To ensure that the contours of the arteries are always visible, the edges of the coronary arteries are emphasized around parts that are rendered with low opacity.

Apart from occlusions caused by the coronary arteries, the individual parts of the heart can also cause occlusions. This is most obvious with the right ventricle which completely occludes the septum, as is shown in Figure 11c. The septum is not directly fed by the coronary arteries. Therefore infarction occurs more easily in this area. Since most parts of the polygonal heart model, including the right ventricle, 


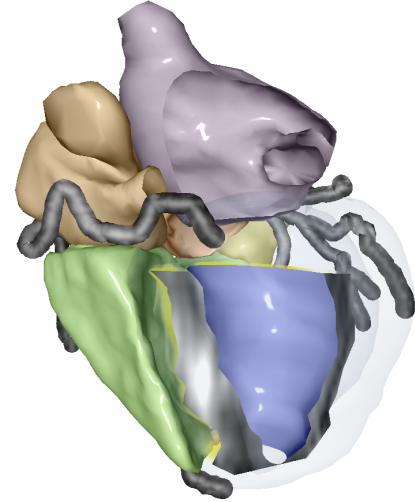

(a)

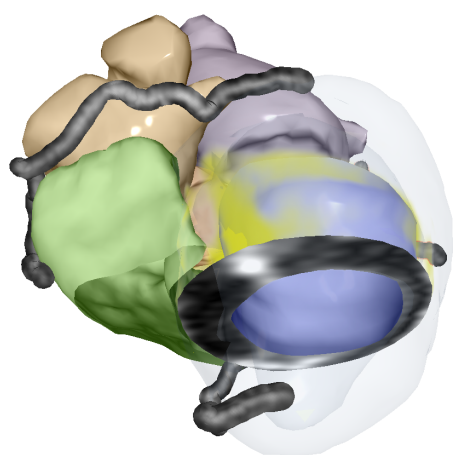

(b)
Figure 12: Cutting plane in (a) long axis orientation and (b) short axis orientation both with LE data reformatted along the plane. The white spots represent scar.

offer only context information, simple visibility reduction techniques are sufficient. Figure 11d illustrates a rendering with a reduced opacity for the right ventricle, causing the scar in the septum to become visible.

\subsection{Visualizing Slice Data}

The CoViCAD framework also allows for the exploration of the MRI slice data of both scans in an anatomical context. This is achieved by adding a cutting plane to the anatomical view. Figure 12 demonstrates this concept. The part of the heart model and the LE data that are in front of the cutting plane are clipped. Along the portion of the cutting plane inside the left ventricular myocardium either the WH or LE data is reformatted. This allows for the observation of the slice data in the myocardium without occlusions, while the rest of the heart model still provides anatomical context. The LE data is also rendered behind the cutting plane. Depending on the viewpoint and orientation of the cutting plane, this can still provide information on the distribution of scar.

To a certain degree, the use of a cutting plane also allows the visualization of the transmurality of scar. However, an area exhibiting scar is commonly first identified in the anatomical view without using a cutting plane. Using the anatomical view to visualize the transmurality of that particular area of scar requires a different viewpoint. Manually positioning the cutting plane and adjusting the viewpoint is generally too time consuming and too complex for the user.

To simplify the specification of the location and orientation of the cutting plane, we offer two basic orientations. The first orientation, shown in Figure 12a, starts with the typical four-chamber view of the heart. Here the plane is parallel to both the short and long axes. The plane can then only be rotated around the long axis. A preset rotation offset is offered that also gives a two-chamber view. The second orientation, shown in Figure 12b, corresponds to the standard short-axis view, where the plane is orthogonal to the long axis. Interaction is restricted to translating the plane along the long axis. These restrictions make specifying the location and orientation of the cutting plane much simpler and more intuitive.

\subsection{Integration of Diverse Rendering Techniques}

The heart model is rendered as a polygonal mesh, the LE data is rendered using direct volume rendering and the coronary artery tree is rendered either as a set of solid tubes, as a MIP or as a CPR. Due to the difference in rendering methods, these components are first all rendered to individual images, while depth information is preserved. For volume rendering techniques such as DVR and MIP where depth is not clearly defined, the depth of the first sample with a non-zero opacity is used. This proves to be an acceptable solution in practice. To construct the final anatomical view, these images are blended together. The depth information of each image is taken into account to preserve correct depth relations. The coronary ghosting takes advantage of this approach. It is implemented by controlling the opacity of the layer with the coronary artery rendering by the opacity in the layer with the LE rendering. For the ghosting the edges of the coronary arteries are rendered using an edge detection operator on the depth information.

\section{Interactive Exploration with Linked Views}

An important requirement of a clinical software assistant is that it should be intuitive, comprehensive and time-efficient. With most current systems, a lot of time-consuming interaction is required. Our system aims to reduce the required amount of interaction to a minimum. The various views on the data present in our system are intended to be used together. This helps maintain a relation between the different views and the properties expressed.

\subsection{Interactive Navigation}

The VBEP serves well for visualizing the overall distribution of scar. Due to the deformations introduced by the unfolding, proportions are however not optimally preserved. Therefore the size of scar is better perceived in the anatomical view. The latter also provides a more detailed, 3D anatomical context. To easily relate a region of interest between these two views we provide the user with a navigation cursor. It can be used to specify a region of interest on the VBEP. The corresponding region in the anatomical view is subsequently highlighted. This is demonstrated in Figure 13. It provides a simple but effective linked navigation between the two views.

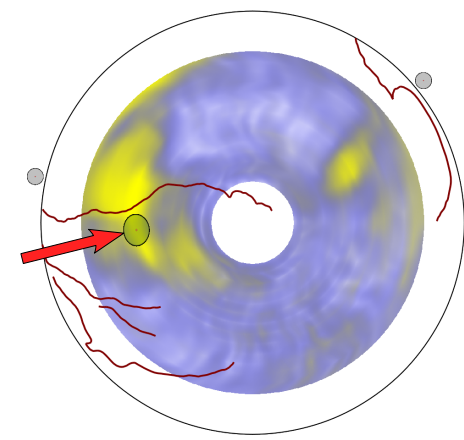

(a)

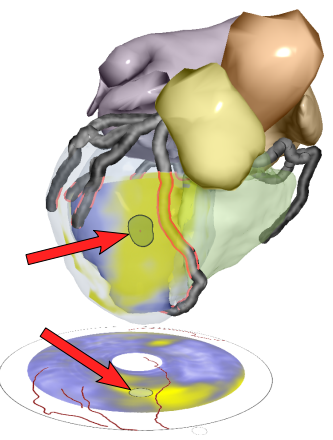

(b)
Figure 13: Global cursor for linking two views: (a) shows a VBEP with the global cursor (indicated with the red arrow) highlighting an area of scar; (b) shows the same region highlighted in the anatomical view. The viewpoint of the anatomical view is controlled by the position of the cursor.

\subsection{Standard Views}

A number of standard views on the heart is used in clinical practice. The most common are the short-axis, two-chamber long-axis and fourchamber long-axis views. These imaging planes are often used for data acquisition. The long and short axes provide a better view of the heart than the standard patient axes.

We have applied this knowledge to the viewpoint control of the anatomical view. The four-chamber long-axis view as seen in Figure 9 provides a good starting viewpoint for the heart. An advantage of using standard views as an initial viewpoint estimation is that it can compensate for the differences in orientation of the heart between patients. Due to occlusion issues the other standard views are more useful in combination with a cutting plane. The standard views are in fact originally intended for the exploration of the inner parts of the heart. As mentioned in section 5.3, we also restrict the orientation and interaction possibilities of the cutting plane according to these standard views. This makes interacting with the cutting plane more intuitive and time-efficient.

\subsection{Automatic Viewpoint Control}

Interactive specification of orientation in $3 \mathrm{D}$ visualizations is a timeconsuming task and one of the reasons most such visualizations still 
lack medical acceptance. Therefore we provide an automatic computation of the orientation of the anatomical view based on a region of interest specified in the VBEP. Once the region of interest is specified, the corresponding region on the epicardium is calculated. The viewpoint of the anatomical view is then configured such that it corresponds to the vector from the center of the region and a point located on the long axis, exactly in the middle between the aortic valve and the apex. The latter point acts as the rotation center of the model. This approach is similar to the work of Viola et al. [14], although it employs a more restrictive and much simpler viewpoint specification.

One step further is to automatically create case-specific views without the specification of a region of interest. Using the scar classification function specified by the user, regions in the VBEP that exhibit scar can automatically be calculated. For each of these areas, an accompanying anatomical view can be generated. In this case the regions of interest are automatically identified. In a typical work-flow scenario a clinician would step through the automatically generated anatomical views, discarding those where the tissue in focus was falsely classified as scar. This could prove to reduce the required amount of interaction and the duration of the analysis. We have however not yet explored the full potential of this approach.

\section{Conclusions and Future Work}

We have presented CoViCAD; a comprehensive medical visualization framework for the diagnosis of patients with CAD using segmented WH and LE MRI data. Our framework presents the data both in a schematic way and in a $3 \mathrm{D}$ view with anatomical context. The former presentation mainly visualizes the overall distribution and transmurality of scar, while the latter serves for the assessment of size and location of scar with relation to the myocardial and coronary anatomy. Our implementation takes advantage of the capabilities of current graphics hardware to offer interactively explorable visualizations. We tested our framework on an Nvidia GeForce 9750 GX2 and obtained more than 20 frames per second for all our visualizations.

By introducing the VBEP we have extended the BEP, an existing, clinically accepted visualization concept. The VBEP preserves transmurality information, removes discontinuities and adds coronary anatomical context. The transmurality of scar is visualized in a rectangular unfolding of the myocardium, which is closely related to the VBEP. Both these visualizations are novel in this application area.

The $3 \mathrm{D}$ visualization of scar in an anatomical context restores the relation to the myocardial and coronary anatomy. Regions of scar can be related to coronary arteries, which can be examined for a possible stenosis using various representations. This allows the clinician to relate the cause (stenosis) to result (scar). The essential part of our approach is that it offers a visual examination of the coronary anatomy in relation to scar. Currently the resolution of the coronary arteries in WH MRI scans is not sufficient for accurate diameter measurements. Moreover a stenosis can even be hard to find through visual inspection. A visualization in relation to scar therefore helps to find a stenosis by focusing on particular parts of the coronary tree. The VBEP also allows relating scar to coronary arteries. Due to these advantages, combined with the increased accuracy and additional information on transmurality of the VBEP compared to the BEP, we hypothesize that our framework will lead to a more accurate and efficient diagnosis of patients with CAD.

The visualization of scar in an anatomical context is also applicable to patients that suffer from an electrical disorder that causes part of their heart to beat asynchronously. These patients can be treated with cardiac resynchronization therapy, a procedure in which a pacemaker with multiple wires is implanted into the patient. It is important that none of these wires are implanted in areas of scar, since scar has a reduced conductivity and thus would void their functionality. A 3D visualization is in this case better understandable than a series of 2D images. It relates better to the anatomy visible during surgery. This is most likely also the reason that there is an increasing demand from our medical partners, especially from cardiologists, for 3D visualizations like the anatomical view we presented.

The integration of multiple data sources and rendering techniques in our anatomical view is more comprehensive than has been presented up to now. Much thought has been spent on the interaction between the different views in order to keep the amount of required interaction as low as possible. The result is an intuitive and comprehensive analysis tool for diagnosing patients with CAD.

Although we have not yet performed a thorough medical evaluation of our techniques, CoViCAD has been developed in tight cooperation with our medical partner. Company experts in close contact with medical heart specialists provided the required specifications and steering feedback to accommodate the needs of the domain experts.

\section{Acknowledgements}

This work was performed in the scope of the COMRADE project funded by Philips Medical Systems, Best, The Netherlands. The datasets were provided by Hyogo BHC at Himeji and the Tokyo Metro Police Hospital.

\section{References}

[1] M. D. Cerqueira, N. J. Weissman, V. Dilsizian, A. K. Jacobs, S. Kaul, W. K. Laskey, D. J. Pennell, J. A. Rumberger, T. Ryan, and M. S. Verani. Standardized myocardial segmentation and nomenclature for tomographic imaging of the heart. Circulation, 105:539-542, 2002.

[2] O. Ecabert, J. Peters, and J. Weese. Modeling shape variability for full heart segmentation in cardiac computed-tomography images. In Proc. SPIE, volume 6144, pages 1199-1210, 2006.

[3] C. Everitt. Interactive order-independent transparency. Technical report, nVidia Corporation, 2001.

[4] M. Hadwiger, C. Berger, and H. Hauser. High-quality two-level volume rendering of segmented data sets on consumer graphics hardware. In Proc. IEEE Visualization, pages 301-308, 2003.

[5] A. Kanitsar, D. Fleischmann, R. Wegenkittl, P. Felkel, and E. Gröller. CPR - Curved Planar Reformation. In IEEE Visualization, pages 37-44, 2002.

[6] Y. J. Kim, J.-S. Seo, B. W. Choi, K. O. Choe, Y. Jang, and Y.-G. Ko. Feasibility and diagnostic accuracy of whole heart coronary MR angiography using free-breathing 3D balanced turbo-field-echo with SENSE and the half-fourier acquisition technique. Korean Journal of Radiology, 7:235242, 2006.

[7] C. Kuehnel, A. Hennemuth, S. Bock, S. Oeltze, T. Boskamp, S. Krass, B. Preim, and H.-O. Peitgen. New software assistants for cardiovascular diagnosis. In GI-Workshop "Softwareassistenten - Computerunterstützung für die medizinische Diagnose und Therapieplanung” within the GI annual conference, pages 491-498, 2006.

[8] C. Lorenz, S. Renisch, T. Schlathoelter, and T. Buelow. Simultaneous segmentation and tree reconstruction of the coronary arteries in MSCT images. In Proc. SPIE, volume 5031, pages 167-177, 2003.

[9] C. Lorenz and J. von Berg. A comprehensive shape model of the heart. Medical Image Analysis, 10:657-670, 2006.

[10] N. M. Noble, D. L. Hill, M. Breeuwer, and R. Razavi. The automatic identification of hibernating myocardium. MICCAI, 0:890-898, 2004.

[11] S. Oeltze, A. Kuß, F. Grothues, A. Hennemuth, and B. Preim. Integrated visualization of morphologic and perfusion data for the analysis of coronary artery disease. In Proc. EuroVis, pages 131-138, 2006.

[12] O. Pereztol-Valdes, J. Candell-Riera, C. Santana-Boado, J. Angel, S. Aguade-Bruix, J. Castell-Conesa, E. V. Garcia, and J. Soler-Soler. Correspondence between left ventricular 17 myocardial segments and coronary arteries. European Heart Journal, 26:2637-2643, 2005

[13] M. Straka, M. Červeňanský, A. L. Cruz, A. Köchl, M. Šrámek, E. Gröller, and D. Fleischmann. The VesselGlyph: Focus \& context visualization in CT-angiography. In Proc. IEEE Visualization, pages 385-392, 2004.

[14] I. Viola, M. Feixas, M. Sbert, and M. E. Gröller. Importance-driven focus of attention. In Proc. IEEE Visualization, pages 933-940, 2006. 